\title{
Two-dimensional Model of an Electromagnetic Layer for the Mitigation of Communications Blackout
}

\author{
Minkwan $\mathrm{Kim}^{1}$ \\ University of Michigan, Ann Arbor, MI, 48109 \\ Michael Keidar ${ }^{2}$ \\ George Washington University, Washington, D.C, 20052 \\ and \\ Iain D. Boyd ${ }^{3}$ \\ University of Michigan, Ann Arbor, MI, 48109
}

\begin{abstract}
During hypersonic, the shock heated air generates a weakly ionized plasma layer around a vehicle. Since the created plasma layer has a high plasma number density, the vehicle has a communication problem known as radio blackout. Solving radio blackout is an important issue for safety of the vehicle, catastrophe analysis, and mission success. In order to solve radio blackout, we propose to manipulate a plasma number density. This paper describes studies of manipulating a flowing plasma with electric and magnetic fields. We suggest a two-dimensional model of an ExB layer which helps to determine the optimal configuration of the ExB layer and location of an antenna. The suggested numerical model uses a hydrodynamic MHD approximation and it is solved using a finite volume method with a Riemann solver. In this paper, we demonstrate that an applied ExB layer can manipulate plasma density in a specific region. The manipulated plasma reduces radio wave attenuation in a plasma layer and provides the possibility for communication during radio blackout.
\end{abstract}

\section{Nomenclature}

$\mathbf{B}=$ magnetic field, $[\mathrm{T}]$

$\mathbf{E} \quad=$ electric field, $[\mathrm{V} / \mathrm{m}]$

$f_{\text {radio }}=$ radio wave frequency, $[\mathrm{Hz}]$

$f_{p} \quad=$ plasma frequency, $[\mathrm{Hz}]$

$\mathbf{J}=$ current density, $\left[\mathrm{A} / \mathrm{m}^{2}\right]$

$M_{e} \quad=$ electron mass, $0.911 \times 10^{-30}[\mathrm{~kg}]$

$M_{i} \quad=$ ion mass, $[\mathrm{kg}]$

$n \quad=$ plasma number density, $\left[\mathrm{m}^{-3}\right]$

$p \quad=$ total plasma pressure, $\left[\mathrm{N} / \mathrm{m}^{2}\right]$

$\mathbf{R}_{e} \quad=$ friction force for the electrons

$\mathbf{R}_{i} \quad=$ friction force for the ions

$\mathrm{R}_{\mathrm{M}} \quad=$ magnetic Reynolds number

$T_{e} \quad=$ electron temperature, $[\mathrm{eV}]$

$T_{i} \quad=$ ion temperature, $[\mathrm{eV}]$

$\mathbf{V} \quad=$ plasma flow velocity, $[\mathrm{m} / \mathrm{s}]$

$\beta_{e} \quad=$ Hall parameter

$\lambda=$ magnetic field stream function

\footnotetext{
${ }^{1}$ Graduate student, Department of Aerospace Engineering, minkwan@umich.edu, AIAA Student Member

${ }^{2}$ Assistant Professor of Engineering and Applied Science, Department of Mechanical and Aerospace Engineering, keidar@gwu.edu, AIAA Associate Fellow

${ }^{3}$ Professor, Department of Aerospace Engineering, iainboyd@umich.edu, AIAA Associate Fellow
} 


$\begin{array}{lll}\mu_{i} & =\text { ion mobility } \\ \sigma & =\text { DC conductivity, }[\mathrm{S} / \mathrm{m}] \\ \tilde{\sigma} & =\text { electrical conductivity tensor } \\ \phi & =\text { plasma potential, }[\mathrm{V}] \\ \text { Subscripts } & \\ e & =\text { electron } \\ i & =\text { ion } \\ o & =\text { initial condition }\end{array}$

\section{Introduction}

$\mathrm{T}$ HE communication or radio blackout problem is an important issue for hypersonic vehicles. When a vehicle flies with hypersonic velocity, a plasma layer is created as a direct consequence of conversion of kinetic energy of the vehicle into thermal and internal energy of the surrounding medium via a shock wave. ${ }^{1}$ Within the shock wave, a plasma layer is formed when the temperature is high enough to excite the gas molecule internal energy modes up to the point where dissociation and ionization reactions occur. The created plasma layer usually has an electron number density of $10^{17}$ to $10^{20} \mathrm{~m}^{-3}$, and it may cause negative effects on a vehicle's operation, particularly for its communications. ${ }^{2}$ When the plasma frequency of the plasma layer around a vehicle exceeds the radio wave frequency used for communications: $f_{\mathrm{p}}>f_{\text {radio, }}$, the plasma layer reflects or attenuates radio waves which are transmitted from or to a vehicle. ${ }^{3}$ Therefore communication is temporarily interrupted, which is known as radio/communication blackout. For re-entry hypersonic vehicles, radio blackout typically lasts several minutes, depending on the angle of re-entry and the particular trajectory. ${ }^{2,4}$

It is extremely important to develop strategies for propagating telemetry during the radio blackout of hypersonic flight. When the radio/communication blackout happens, vehicles lose voice communication, data telemetry, and GPS navigation. ${ }^{4}$ This is critical for vehicle safety because the vehicles cannot receive any guidance information from ground stations or GPS satellites. The vehicle's safety is compromised when it travels hundreds of miles during a few minutes of blackout without any guidance information such as GPS navigation or voice communication. ${ }^{5}$ For example, the Soyuz TMA re-entry vehicle experiences about 10 minutes of radio blackout, enough time for it to travel several thousand miles without guidance from a ground station or GPS satellite. ${ }^{2}$ Radio blackout also makes catastrophe analysis impossible, eliminating a critical factor for understanding and preventing reentry accidents. In the space shuttle Colombia disaster, telemetry was lost prior to disintegration due to radio blackout. ${ }^{6}$ When the telemetry was recovered from the radio blackout, there was little available data at the ground station to assist in determining the cause of the disaster. In addition, radio blackout plays a significant role in mission success. For an unmanned vehicle, a blackout makes it lose control or guidance from a ground station. In this case, continuous and real-time telemetry determines whether the vehicle succeeds in a mission or not.

During the last 50 years, a number of approaches have been suggested to solve the problem of radio blackout during re-entry or hypersonic flight. ${ }^{2,4}$ They include aerodynamic shaping, quenchant injection, high frequencies, and Raman scattering ${ }^{4}$. The suggested approaches have technical or practical limitations such as cost, system weight, and aerodynamic performance, so they can only apply in specific cases. For example, the space shuttle solved the radio blackout problem by using NASA's Tracking and Data Relay Satellite (TDRS) system, but even the TDRS is not a general solution for the radio blackout problem. Since the TDRS requires the tail end of the vehicle to have less ionization than the front of the vehicle to provide a hole through which communications with the TDRS can be maintained, it cannot be used with ballistic re-entry vehicles or with powered air-breathing lifting vehicles. The TDRS also cannot be used in Mars entry missions because Mars has no communication-satellite system.

Previously, we suggested a strategy for propagating telemetry through a plasma layer in a feasible and reliable manner. ${ }^{2,7}$ Our re-entry telemetry scheme expands upon the magnetic window method via the addition of electric fields to increase the density reduction possible for a given magnetic-field strength. The magnetic window method is one of the most promising methods where a strong DC magnetic field is used to allow radio-wave propagation through the plasma layer. ${ }^{4}$ However, this technique is limited by the required magnetic field strength. A reasonable magnetic field strength is up to $0.15 \mathrm{~T}$ in terms of acceptable weight and volume for re-entry vehicles but an approximately $1 \mathrm{~T}$ magnetic field is required to penetrate the plasma layer by using the magnetic window method. ${ }^{8}$ The limitation of the magnetic-window method can be improved upon by the addition of an electric field. The applied electric-field accelerates the plasma temporarily over the antenna, reducing the local density to allow communication. As shown in Fig. 1, the reduced plasma density can create a "window" in the re-entry plasma layer through which radio waves can be transmitted and received. However, the use of an electric field alone is 
impractical due to shielding of the electrodes by sheath effects. At high densities, the sheath which forms around the electrodes can have a thickness of millimeters or less. The electrode-shielding problem can be decreased by a magnetic field.

In previous work, we demonstrated the effectiveness of the electromagnetic field with a two-dimensional simulation. The results showed that the applied $\mathrm{ExB}$ layer can effectively reduce the plasma number density out to approximately $3 \mathrm{~cm}$ from the surface. ${ }^{2}$ These are very promising results for mitigating the radio blackout problem, because the plasma density peak in the RAM-C hypersonic re-entry flight is at about $1-2 \mathrm{~cm}$ from the vehicle surface. ${ }^{9,10}$ Our two-dimensional model used a constant one-dimensional magnetic field, although practically it is difficult to create a constant one-dimensional magnetic field. During the process of creating the window, the plasma density reduction is significantly affected by the magnetic field configuration. Therefore the overall performance of the electromagnetic scheme for reentry telemetry is considerably affected by the magnetic-field configuration. A two-dimensional magnetic-field model can help to predict the effectiveness of the electromagnetic ExB layer as the re-entry telemetry scheme.

In this paper, we use a two-dimensional magnetic-field configuration which is based on experimental data and demonstrate the effectiveness of the two-dimensional magnetic-field model in comparison to the one-dimensional magnetic-field model. This will be seen as an effective approach to solving the blackout problem. In Sec. II, we describe a physical model of an ExB layer with a two-dimensional magnetic-field. The numerical method and boundary conditions are described in Sec. III. Section IV shows numerical results of the two-dimensional ExB layer model and evaluates the results in terms of blackout mitigation. Conclusions are formulated in Sec. V.

\section{Two-dimensional Magnetic Field Modeling}

The magnetic field is one of the most important parameters affecting plasma-density reduction in the ExB layer. In order to investigate the effectiveness of the magnetic-field configuration, a variable magnetic-field model is needed. In this study, we model the two-dimensional variable magnetic-field of the ExB layer. The governing equations for the ExB layer are composed of two parts which are the flow field and the electric field.

\section{A. Plasma flow description}

We previously developed a two-dimensional MHD model of an ExB layer. ${ }^{1}$ This model is based on a twodimensional steady-state fluid plasma model with the following general assumptions ${ }^{1}$ :

1) The ExB layer is quasi-neutral.

2) The neutrals are at rest.

3) There is no ionization in the ExB layer.

4) The electron temperature, $T_{e}$, is constant at $5 \mathrm{eV}$.

5) The ions are cold in hypersonic flow, $T_{i} \approx T_{e}$.

6) The ion mobility, $\mu_{i}$, is determined by ion-neutral collisions.

These assumptions are physically reasonable for this application. First, quasi-neutrality is consistent with measured data from re-entry vehicles. ${ }^{10}$ The measured data shows the Debye length is typically on the order of $10^{-6} \mathrm{~m}$ so the sheath region is very small compared with the plasma region. Although strictly speaking the neutrals are not at rest with an incoming hypersonic flow condition, the stationary neutral assumption will maximize the effect of the ionneutral drag term in the model, thus rendering our calculations for a worst-case scenario. Furthermore the boundary layer around the vehicle results in relatively slow neutral velocity compared to the vehicle velocity, and it is in this stagnation layer that the plasma density to be mitigated is highest. In the ExB layer model, we concentrate on a hypersonic boundary layer. The plasma is already created by associative and electron-impact ionization which are the two production mechanisms of electrons in a hypersonic flow. ${ }^{11}$ Therefore, we do not need to consider an additional plasma creation by ionization. Therefore, the two-dimensional steady-state ExB layer model can be described by:

$$
\begin{gathered}
\nabla \cdot\left(n_{i} \mathbf{V}\right)=0 \\
M_{i} n_{i}\left(\mathbf{V}_{i} \cdot \nabla \mathbf{V}_{i}\right)=e n_{i}\left(\mathbf{E}+\mathbf{V}_{i} \times \mathbf{B}\right)-\nabla p_{i}+\mathbf{R}_{i} \\
\nabla \cdot\left(n_{e} \mathbf{V}_{e}\right)=0
\end{gathered}
$$




$$
0=-e n_{e}\left(\mathbf{E}+\mathbf{V}_{e} \times \mathbf{B}\right)-\nabla p_{e}+\mathbf{R}_{e}
$$

where $\mathbf{R}_{i}$ is the friction force for the ions and $\mathbf{R}_{e}$ is the friction force for electrons. Equations (1) and (2) are mass and momentum conservation for ions, respectively. Equations (3) and (4) are mass and momentum conservation for electrons, respectively.

We consider a quasi-neutral plasma with one species of ions so that

$$
n_{i}=n_{e} \equiv n
$$

In order to simplify Eqs. (1)-(4), we employ the plasma velocity $\mathbf{V}$ and the electric current $\mathbf{J}$ defined by

$$
\begin{gathered}
\mathbf{V}=\frac{M_{i} \mathbf{V}_{i}+M_{e} \mathbf{V}_{e}}{M_{i}+M_{e}} \\
\mathbf{J}=e n\left(\mathbf{V}_{i}-\mathbf{V}_{e}\right)
\end{gathered}
$$

Due to $M_{e} \ll M_{i}$, the ion velocity becomes the plasma velocity

$$
\mathbf{V}_{i} \approx \mathbf{V}
$$

From Eq. (8), the ions mass-conservation equation, Eq. (1), gives the mass continuity equation for the plasma flow.

$$
\nabla \cdot(n \mathbf{V})=0
$$

The plasma momentum equation is obtained from summation of momentum equations, Eqs. (2) and (4).

$$
M_{i} n(\mathbf{V} \cdot \nabla \mathbf{V})=(\mathbf{J} \times \mathbf{B})-\nabla p+\mathbf{R}
$$

where we have made use of the total plasma pressure $p=p_{i}+p_{e}$. Therefore, Eqs. (9) and (10) describe the plasma flow in an ExB layer. In Eq. (10), the $\mathbf{J} \times \mathbf{B}$ term is the Lorenz Force accelerating the plasma.

\section{B. Two-dimensional magnetic field model}

The ExB layer model, Eqs. (9) and (10), needs a description for the current density, J. From subtraction of mass conservations of ions and electrons, Eqs. (1) and (3), we obtain the current density conservation as

$$
\nabla \cdot \mathbf{J}=0
$$

The current density is calculated from the generalized Ohm's law. The generalized Ohm's law can be expressed in a matrix form as follows:

$$
\mathbf{J}=\tilde{\sigma}(\mathbf{E}+\mathbf{V} \times \mathbf{B})
$$

where $\tilde{\sigma}$ is the conductivity tensor:

$$
\tilde{\sigma}=\frac{\sigma}{1+\beta_{e}^{2}}\left[\begin{array}{ccc}
1+\beta_{x}^{2} & \beta_{x} \beta_{y} & \beta_{x} \beta_{z} \\
\beta_{y} \beta_{x} & 1+\beta_{y}^{2} & \beta_{y} \beta_{z} \\
\beta_{z} \beta_{x} & \beta_{z} \beta_{y} & 1+\beta_{z}^{2}
\end{array}\right]
$$


where: $\beta_{x}=B_{x} \frac{\beta_{e}}{B}, \beta_{y}=B_{y} \frac{\beta_{e}}{B}, \beta_{z}=B_{z} \frac{\beta_{e}}{B}, \beta_{e}$ is the Hall parameter, and $\sigma$ is the DC conductivity.

The conductivity tensor, Eq. (13), can be simplified for a two-dimensional magnetic-field configuration. A schematic of the two-dimensional magnetic-field model of the electromagnetic layer is shown in Fig. 2. In this study, we consider the magnetic field has only $\mathrm{x}$ and $\mathrm{z}$ direction components, $B_{x}, B_{z}$. In this case, the conductivity tensor becomes

$$
\tilde{\sigma}=\frac{\sigma}{1+\beta_{e}^{2}}\left[\begin{array}{ccc}
1+\beta_{x}^{2} & 0 & \beta_{x} \beta_{z} \\
0 & 1 & 0 \\
\beta_{z} \beta_{x} & 0 & 1+\beta_{z}^{2}
\end{array}\right]
$$

Thus the component forms of the current density become

$$
\left(\begin{array}{l}
J_{x} \\
J_{y} \\
J_{z}
\end{array}\right)=\frac{\sigma}{1+\beta_{e}^{2}}\left[\begin{array}{ccc}
1+\beta_{x}^{2} & 0 & \beta_{x} \beta_{z} \\
0 & 1 & 0 \\
\beta_{z} \beta_{x} & 0 & 1+\beta_{z}^{2}
\end{array}\right] \cdot\left(\begin{array}{c}
E_{x} \\
V_{z} B_{x}-V_{x} B_{z} \\
E_{z}
\end{array}\right)
$$

From the current-density conservation, Eq. (11), with the usual definition of an electric field, Eq. (15) becomes

$$
\nabla \cdot(\tilde{\sigma} \nabla \phi)=\nabla \cdot(\tilde{\sigma}(\mathbf{V} \times \mathbf{B}))
$$

Therefore we obtain a Poisson-like equation for the potential distribution from Eq. (16).

$$
\begin{aligned}
& \left(\frac{1+\beta_{e, z}{ }^{2}}{1+\beta_{e, x}{ }^{2}+\beta_{e, z}{ }^{2}} \sigma\right) \frac{\partial^{2} \phi}{\partial x^{2}}+\left(\frac{1+\beta_{e, x}{ }^{2}}{1+\beta_{e, x}{ }^{2}+\beta_{e, z}{ }^{2}} \sigma\right) \frac{\partial^{2} \phi}{\partial z^{2}}+\left(\frac{2 \beta_{e, x} \beta_{e, z}}{1+\beta_{e, x}{ }^{2}+\beta_{e, z}{ }^{2}} \sigma\right) \frac{\partial^{2}}{\partial x \partial z} \phi \\
& +\left\{\frac{\partial}{\partial x}\left(\frac{1+\beta_{e, z}{ }^{2}}{1+\beta_{e, x}{ }^{2}+\beta_{e, z}{ }^{2}} \sigma\right)+\frac{\partial}{\partial x}\left(\frac{\beta_{e, x} \beta_{e, z}}{1+\beta_{e, x}{ }^{2}+\beta_{e, z}{ }^{2}} \sigma\right)\right\} \frac{\partial \phi}{\partial x} \\
& +\left\{\frac{\partial}{\partial x}\left(\frac{\beta_{e, x} \beta_{e, z}}{1+\beta_{e, x}{ }^{2}+\beta_{e, z}{ }^{2}} \sigma\right)+\frac{\partial}{\partial x}\left(\frac{1+\beta_{e, x}{ }^{2}}{1+{\beta_{e, x}}{ }^{2}+\beta_{e, z}{ }^{2}} \sigma\right)\right\} \frac{\partial \phi}{\partial z}=0
\end{aligned}
$$

Equation (17) is a non-linear $2^{\text {nd }}$ order Elliptic PDE. Solving Eq. (17) gives the potential distribution of an ExB layer.

\section{Numerical method and boundary conditions}

The two-dimensional ExB layer MHD model, Eqs.(9), (10), and (17), is solved numerically with an iterative scheme. The solution begins with an assumed initial potential distribution, $\varphi^{0}$ which is obtained by solving the Poisson equation. Equations (9) and (10) are solved by using the finite volume method with the Harten-Lax-van Leer contact wave (HLLC) Riemann solver ${ }^{12}$ to give plasma number density and velocity. The obtained plasma number density and velocity distributions are used to calculate the new potential distribution. The potential distribution is obtained from solving Eq. (17). The alternating direction implicit (ADI) method is used to solve Eq. (17) for the two-dimensional magnetic-field model with Poisson-like equation. Since the coefficient of the last two terms of Eq. (17) 


$$
\begin{gathered}
\left\{\frac{\partial}{\partial x}\left(\frac{1+\beta_{e, z}{ }^{2}}{1+\beta_{e, x}{ }^{2}+\beta_{e, z}{ }^{2}} \sigma\right)+\frac{\partial}{\partial z}\left(\frac{\beta_{e, x} \beta_{e, z}}{1+\beta_{e, x}{ }^{2}+\beta_{e, z}{ }^{2}} \sigma\right)\right\} \frac{\partial \phi}{\partial x} \\
\text { and }\left\{\frac{\partial}{\partial x}\left(\frac{\beta_{e, x} \beta_{e, z}}{1+\beta_{e, x}{ }^{2}+\beta_{e, z}{ }^{2}} \sigma\right)+\frac{\partial}{\partial z}\left(\frac{1+\beta_{e, x}{ }^{2}}{1+\beta_{e, x}{ }^{2}+\beta_{e, z}{ }^{2}} \sigma\right)\right\} \frac{\partial \phi}{\partial z}
\end{gathered}
$$

may be much larger than other terms, we must carefully discretize Eq. (17). Therefore the upwind discretization scheme is applied. The new potential distribution provides a new electric field for the next iteration. After several iterations, all variables converge and we obtain a steady-state solution with sufficient accuracy.

Figure 3 shows boundary conditions which are applied in the two-dimensional ExB layer MHD model. The inflow boundary condition is applied for the left side. It uses the initial plasma number density and the constant neutral number density. In this case, the bulk-plasma velocity has only an x-direction component. The right and upper side boundaries use the outflow boundary condition and no potential variation across the boundary is assumed. The bottom boundary uses the dielectric boundary condition. Due to the dielectric boundary condition, no ions are neutralized at the dielectric wall, which means that the wall fully absorbs ions. An applied potential boundary-condition at the electrodes is shown in Fig. 4. It uses a linear distribution between the two electrodes and an exponential distribution beyond the electrodes for numerical stability.

An applied two-dimensional magnetic-field is generated by an electromagnet. In the present study, we assume the magnetic Reynolds number of a hypersonic flow field is much smaller than unity. The magnetic Reynolds number, $\mathrm{R}_{\mathrm{M}}$, is a measure of the coupling between the motion of the magnetic field line and the motion of the conducting fluid. When the magnetic Reynolds number is much smaller than unity, the magnetic field is not greatly affected by the fluid motion. ${ }^{13}$ It means the induced magnetic field by current is negligible compared with the imposed magnetic field. ${ }^{14}$ Due to the small magnetic Reynolds number assumption, we can neglect the induced magnetic field. Therefore the generated two-dimensional magnetic field is assumed to be entirely determined by the applied electromagnet and to be unaffected by its discharge.

Figure 5 shows the configuration of an applied two-dimensional magnetic-field in this study. The applied magnetic field is calculated using a Poisson solver with measured magnetic-field data. In the MHD limit, a magnetic field satisfies a divergence free condition:

$$
\nabla \cdot \mathbf{B}=0
$$

A magnetic field stream function, $\lambda$, is defined as

$$
B_{x}=\frac{\partial}{\partial x} \lambda \text { and } B_{z}=\frac{\partial}{\partial z} \lambda
$$

Equation (18) becomes a Poisson equation with a magnetic field stream function, $\lambda$.

$$
\nabla^{2} \lambda=0
$$

The boundary conditions of Eq. (19) are used from a magnetic-field data measured in the Plasmadynamics and Electric Propulsion Laboratory (PEPL) at the University of Michigan. Solving Eq. (19) gives a magnetic-field distribution for this study. As shown in Fig. 5, the configuration of a magnetic field does not depend on the maximum magnetic-field strength and the maximum magnetic-field strength is given near the two electrodes.

\section{Results}

\section{A. An ExB layer with a two-dimensional magnetic-field}

We first apply our model to a simple configuration that has been studied experimentally. The simple configuration of the ExB layer is shown schematically in Fig. 2. In Fig. 2, an electric field is applied using two electrodes. The anode and cathode are $5 \mathrm{~mm}$ in length and are separated by $4 \mathrm{~cm}$. Under the two electrodes, an electro magnet is located that provides a two-dimensional magnetic field. For the ExB layer simulation, argon is 
used with a constant background neutral pressure and the neutrals are assumed at rest. The detailed initial conditions of the ExB layer are specified in Table 1. In this study, the magnetic field strength indicates the absolute value of the magnetic field at the center between the two electrodes. The plasma number density is normalized by the plasma bulk density, $\mathrm{n}_{0}$ and it is called the plasma density reduction. A strong plasma density reduction indicates a large effect of the applied ExB layer.

The plasma density reduction with the two-dimensional magnetic field is shown in Fig. 6. It shows the strongest plasma density reduction occurs near the cathode. There are also two increased density regions, beyond the anode and the cathode. The increased density region of the cathode is also shown in the one-dimensional constant magnetic field model. ${ }^{2}$ It is primarily caused by the $0 \mathrm{~V}$ potential beyond the cathode. The applied $0 \mathrm{~V}$ potential is quite reasonable because we assume that the area beyond the cathode has a vacuum condition. However, the onedimensional magnetic field model does not have the increased density region near the anode. We can expect this increased region due to the gradient of the applied two-dimensional magnetic-field. Therefore the optimal location of an antenna can be determined from Fig. 6 and it is near the cathode.

As a mitigation scheme, an ExB layer has two important parameters in terms of communication through a plasma layer; plasma density reduction and effectiveness area of an ExB layer. First, the density reduction required to mitigate through a plasma layer is determined base on the peak plasma density and the radio-wave frequency used for communication. As shown in Table 2, various radio-wave frequencies are used for different applications. This means each application requires a different plasma density reduction to solve radio blackout. When the plasma number density is higher than a critical number density, a radio-wave has infinite attenuation in the plasma layer. Therefore we need to reduce the plasma number density at least below the critical number density. ${ }^{2,5,15}$ A required plasma density reduction for each radio-wave frequency is indicated in Fig. 7. Therefore we can set the desired plasma density reduction for each specific communication radio-wave frequency at the peak plasma number density of an ExB layer.

Another important parameter is the thickness of the plasma layer which is effectively manipulated by an ExB layer. It is determined base on the required plasma-density reduction. The region effectively manipulated by an ExB layer should be larger than the thickness of the plasma layer which prevents communication. In order to solve radio blackout, the effectiveness area of an ExB layer can be found from Fig. 8 which shows more detail of the plasma density reduction. For a GPS signal which uses $1.5 \mathrm{GHz}$, the required density reduction is about 0.7 for a $4.2 \times 10^{16}$ $\mathrm{m}^{-3}$ bulk plasma density. Therefore Fig. 8 tells us that the applied ExB layer can effectively reduce the plasma density up to approximately $2 \mathrm{~cm}$ above the surface for solving radio blackout of GPS navigation. When the thickness of a plasma layer is smaller than $2 \mathrm{~cm}$, the created the "window" can penetrate the plasma layer for GPS communication. For the RAM-C flight test, the thickness of the plasma layer is approximately $1-2 \mathrm{~cm}$ but it depends on the altitude. For the low-altitude case, the plasma layer is thin and has high peak plasma density. At high altitudes, the plasma layer is thicker and the peak plasma density is lower than the low altitude case. Therefore Fig. 9 shows the applied ExB layer has a possibility to create a "window" for GPS navigation.

The configuration of the magnetic field can affect the plasma density reduction of an ExB layer. Previously, we suggested a one-dimensional magnetic-field model of an ExB layer. ${ }^{2}$ Figure 9 shows the two-dimensional magnetic field model predicts lower plasma density reduction than the one-dimensional magnetic-field model. The reason is a difference of magnetic field strength between the two magnetic-field models, as shown in Fig. 10. The strength of the two-dimensional magnetic field configuration decreases with distance from the wall surface. However, the magnetic-field is constant in the one-dimensional case.

Figures 9 also show the applied ExB layer only affects the plasma density near the cathode. It means an ExB layer will not disturb an entire flow. This is an important fact for a practical application because an applied ExB layer gives a window for communication without any effect on a vehicle's aerodynamics.

In order to optimize the system, it is important to understand the effectiveness of the applied magnetic-field in the ExB layer. In a previous study, we already showed that a larger magnetic field gives a stronger plasma density reduction with the one-dimensional ExB layer MHD model. ${ }^{2}$ The two-dimensional ExB layer MHD model gives similar results. Figure 11 shows the plasma density reduction in the ExB layer with several magnetic field strengths. As expected, a larger magnetic field gives a stronger density reduction. However, the maximum magnetic field strength is limited because of the weight of a permanent magnet. Although the higher magnetic field guarantees a larger window for communication, it gives a negative effect on vehicle performance due to a heavy weight.

Figure 12 can be used to optimize the applied magnetic-field strength. It shows the effectiveness of the magnetic field strength for three different models which are the one-dimensional ExB layer simulation model, the twodimensional ExB layer simulation model with one-dimensional constant magnetic field and the two-dimensional ExB layer simulation model with two-dimensional variable magnetic field. As shown in Fig. 12, the plasma density reduction ratio increases with magnetic field strength. However the increase of the plasma density reduction 
becomes smaller at the strong magnetic field condition. The $0.2 \mathrm{~T}$ magnetic field condition gives the maximized plasma density reduction ratio. It is also interesting that all models agree in the case of large magnetic field, stronger than $0.2 \mathrm{~T}$.

The electric field is another parameter that affects the plasma density reduction in the applied ExB layer. In the one-dimensional case, we showed that a higher potential drop gives a stronger plasma density reduction but the maximum applicable potential drop is limited due to an electrode arching. ${ }^{7}$ For the two-dimensional ExB layer MHD model, the effectiveness of the electric field is shown in Fig. 13. It is found from this figure that the density reduction is improved by a higher potential drop. Furthermore, Fig. 13 shows that the change of the plasma density reduction becomes smaller at the high potential drop. Thus, there is an optimum value for the potential drop in order to communicate through a plasma layer.

\section{B. An ExB layer in a hypersonic flow}

In Figs. 6 and $8-13$, the numerical result does not include the generation of a plasma layer so the entire simulation domain is assumed as a plasma layer. Therefore it is necessary to simulate an ExB layer in hypersonic flow in order to consider a realistic plasma layer. For an $\mathrm{ExB}$ layer simulation in a hypersonic flow, we use the OREX vehicle, which was launched by the H-II rocket from Japan to conduct an earth reentry experiment. ${ }^{18}$ OREX is a blunt body reentry vehicle and it has a nearly zero angle of attack in the reentry experiment. Figure 14 shows the simulation domain with a simplified OREX geometry that is composed of a spherical nose with a $1.35 \mathrm{~m}$ radius and a 50-deg sphere cone. Figure 14 also shows the location of the cathode with $5 \mathrm{~mm}$ width and the electromagnet. An anode is grounded on the vehicle so it has a $0 \mathrm{~V}$ potential.

OREX is simulated at the $59.4 \mathrm{~km}$ flight condition with an ExB lay for the plasma manipulation in the boundary layer. The applied magnetic-field configuration is similar to Fig. 5. Table 3 shows inflow conditions and wall temperature of OREX at $59.4 \mathrm{~km}$. The chemical composition of free stream air is assumed to be $79 \% \mathrm{~N}_{2}$ and $21 \%$ $\mathrm{O}_{2}$.

Figure 15 shows the electron number density distribution with the ExB layer. As shown in Fig. 15, the applied ExB layer reduces the electron number density. It shows the plasma density reduction increases with magnetic field strength. The result predicts the highest plasma density reduction when both the electric field and magnetic field are applied. In Fig. 15, there is a plasma density reduction even when there is no applied voltage to the cathode. The density reduction in this case occurs because of the magnetic field. The applied magnetic field can give a current density though there is no electric field. The generated current density is smaller than with the electric field case but it can give a Lorenz force for the plasma acceleration. When the electric field is applied, it gives a higher current density. In this case, the magnetic field helps to maintain a strong electric field. Therefore the electric field improves the plasma density reduction in the hypersonic plasma layer. Figure 15 also shows the plasma returns to its original density past the applied ExB layer. This means the applied ExB layer can manipulate a plasma layer without disturbing the flow field in order to solve the communication blackout problem.

Figure 16 shows the maximum plasma density reduction as a function of applied magnetic-field strength and potential drop. It agrees with Figs. 12 and 13. The applied ExB layer with $0.5 \mathrm{~T}$ and $-500 \mathrm{~V}$ potential drop gives of a plasma density reduction about 0.15 . In this case, the signal attenuation becomes approximately $5 \mathrm{~dB}$ for X-band which was used for OREX reentry telemetry. This demonstrates the possibility of an ExB layer mitigation scheme to solve radio blackout in the hypersonic flight condition.

\section{Conclusion}

We have studied an ExB layer to allow communication through the plasma layer during the radio blackout period. The numerical result shows a significant density reduction in an ExB layer. The application of electric and magnetic fields could therefore allow radio communication through a hypersonic plasma layer. The numerical result also shows the applied ExB layer reduces the plasma density near a cathode. It means the ExB layer mitigation scheme can manipulate plasma in a specific region. The reduced plasma density and area can be controlled by changing magnetic field strength and potential. Therefore the numerical analysis can help to determine an optimal configuration of an ExB layer as a mitigation scheme. In addition to solving radio blackout, the ExB layer mitigation scheme also can be used in other industrial applications such as semiconductor production and positron storage because it can manipulate plasma in a precise area.

\section{Acknowledgments}

This material is based upon work supported by the Department of Energy National Nuclear Security Administration under Award Number NA28614. This report was prepared as an account of work sponsored by an 
agency of the United States Government. Neither the United States Government nor any agency thereof, nor any of their employees, makes any warranty, express or implied, or assumes any legal liability or responsibility for the accuracy, completeness, or usefulness of any information, apparatus, product, or process disclosed, or represents that its use would not infringe privately owned rights. Reference herein to any specific commercial product, process, or service by trade name, trademark, manufacturer, or otherwise does not necessarily constitute or imply its endorsement, recommendation, or favoring by the United States Government or any agency thereof. The views and opinions of authors expressed herein do not necessarily state or reflect those of the United States Government or any agency thereof. The authors wish to thank Charles Jones, David P. Morris, Christopher N. Davis, Kristina M. Lemmer, Alec D. Gallimore, Brian E. Gilchrist, Peter Peterson, Tim Smith, Jonathan Zagel, and Kenneth G. Powell for very useful discussions on this subject.

\section{References}

1 Mazouffre, S., Pawelec, E., Lago, V., da Silve, M., and Dueck, M., "Plasma Formation During high Speed Flights in Upper Layers of The Earth's Atmosphere," AIAA Paper 2002-5272, 2002.

2 Kim, M. ,Keidar, M., Boyd, I. D., “Analysis of an Electromagnetic Mitigation Scheme for Reentry Telemetry Through Plasma,” Journal of Spacecraft and Rockets, Vol. 45, No. 6, 2008, pp. 1223-1229.

3 Rybak, J., and Churchill, R., "Progress in Reentry Communications," IEEE Transactions on Aerospace and Electronic Systems, Vol. 7, No. 5, September 1971, pp. 879-894.

4 Hartunian, R. A., Stewart, G. E., Fergason, S. D., Curtiss, T. J., and Seibold, R. W., "Causes and Mitigation of Radio Frequency (RF) Blackout during Reentry of Reusable Launch Vehicles", The Aerospace Corporation, AEROSPACE REPORT NO. ATR-2007(5309)-1, 2007.

5 Starkey, R. P., "Electromagnetic Wave/Magneto active Plasma Sheath Interaction for Hypersonic Vehicle Telemetry Blackout Analysis", AIAA Paper 2003-4167, June 2003.

6 CAIB (Columbia Accident Investigation Board), "Columbia Accident Investigation Board Report", Vol. 1, Chapter 2, Government Printing Office, Washington, DC, 2003.

7 Keidar, M., Kim, M., and Boyd, I. D., "Electromagnetic reduction of plasma density during re-entry and hypersonic flights," Journal of Spacecraft and Rockets, Vol. 45, No. 3, 2008, pp. 445-453

8 Hartunian, R. A., Stewart, G. E., Curtiss T. J., Fergason, S. D., and Seibold, R. W., "Implications and Mitigation of Radio Frequency Blackout during Reentry of Reusable Launch Vehicles," AIAA Paper 2007-6633, 2007.

9 Jones, W. L. and Cross, A. E., "Electrostatic Probe Measurements of Plasma Surrounding Three 25,000 Foot per Second Reentry Flight Experiments," NASA SP-252, 1970.

${ }^{10}$ Sims, T. E., and Jones, R. F., "Flight measurements of VHF-signal attenuation and antenna impedance for the RAM A-1 slender probe at velocities up to 17,800 feet per second," NASA Tech. Meno., TM X-760, 1963.

${ }^{11}$ Losev, S. A., Makarov, V. N., and Pogoskekyan, M. Yu., "Model of The Physico-Chemical Kinematics Behind The front of a Very Intense Shock Wave in Air," Fluid Dynamics, Vol. 30, No. 2, 1995.

${ }^{12} \mathrm{Li}, \mathrm{S}$., "An HLLC Riemann solver for magneto-hydrodynamics," Journal of Computational Physics., Vol. 203, 2005, pp. 344-357.

${ }^{13}$ Holt, E. H. and Haskell, R. E., "Magneto-Fluid Dynamics," Foundations of Plasma Dynamics, Macmillan Company, New York, Chapter 14.

${ }^{14}$ Gaitonde, D. V., "Simulation of local and global high-speed flow contron with magnetic fields," AIAA Paper 2005-0560, January, 2005.

${ }^{15}$ Morabito, M. M., "The Spacecraft Communications Balckout Problem Encountered during Passage or Entry of Planetary Atmospheres, " IPN Progress Report 42-150, August 15, 2002.

${ }^{16}$ U.S. Dept. of Commerce National Telecommunications and Information Administration, Manual of Regulations and Procedures for Federal Radio Frequency Management, May 2003 ed. (January 2007 Revision), NTIA, Washington, DC, 2007, Chap. 4.

${ }^{17}$ U.S. Dept. of Commerce National Telecommunications and Information Administration, United States frequency allocations: the radio spectrum, Poster, NTIA, Washington, DC, October 2003.

${ }^{18}$ Yamamoto, Y. and Yoshioka, M., "CFD and FEM coupling analysis of OREX Aerothermodynamic Flight Data," AIAA Paper 95-2087, June 1995. 
Table 1. Initial conditions of a simplified ExB simulation.

\begin{tabular}{|c|c|c|c|c|c|}
\hline $\begin{array}{l}\text { Velocity, } \\
\mathrm{V}_{0}[\mathrm{~m} / \mathrm{s}]\end{array}$ & $\begin{array}{l}\text { Initial plasma density, } \\
\qquad \mathrm{n}_{0}\left[\mathrm{~m}^{-3}\right]\end{array}$ & $\begin{array}{c}\text { Gas pressure, } \\
\text { Pn [mTorr] }\end{array}$ & $\begin{array}{c}\text { Electron temperature, } \\
\mathrm{Te}[\mathrm{eV}]\end{array}$ & $\begin{array}{c}\text { Applied } \\
\text { potential drop, } \\
\Delta \varphi[\mathrm{V}]\end{array}$ & $\begin{array}{l}\text { Magnetic field } \\
\text { strength, } \\
\text { B [T] }\end{array}$ \\
\hline 1000.0 & $4.2 \times 10^{16}$ & 1 & 5.0 & -800 & 0.07 \\
\hline
\end{tabular}

Table 2 Commonly used radio wave frequencies and their limits for the maximum plasma density ${ }^{4,16,17}$

\begin{tabular}{crrl}
\hline Band Name & \multicolumn{1}{c}{ Frequency } & Plasma density limit $\left[\mathrm{m}^{-3}\right]$ & \multicolumn{1}{c}{ Example uses } \\
\hline VHF & $30 \sim 300 \mathrm{MHz}$ & $1.2 \times 10^{13} \sim 1.2 \times 10^{15}$ & Aviation communication \\
UHF & $300 \sim 3000 \mathrm{MHz}$ & $1.2 \times 10^{15} \sim 1.2 \times 10^{17}$ & GPS \\
L band & $1 \sim 2 \mathrm{GHz}$ & $1.3 \times 10^{16} \sim 5.1 \times 10^{16}$ & Military telemetry \\
$\mathrm{S}$ band & $2 \sim 4 \mathrm{GHz}$ & $5.1 \times 10^{16} \sim 2.0 \times 10^{17}$ & Satellite communication, Space shuttle, ISS \\
$\mathrm{X}$ band & $8 \sim 12 \mathrm{GHz}$ & $8.3 \times 10^{17} \sim 1.9 \times 10^{18}$ & Satellite communication, RAM C Test \\
$\mathrm{K}_{\mathrm{u}}$ band & $12 \sim 18 \mathrm{GHz}$ & $1.9 \times 10^{18} \sim 4.1 \times 10^{18}$ & NASA's Tracking Data Relay Satellite (TDRS) \\
$\mathrm{K}_{\mathrm{a}}$ band & $27 \sim 40 \mathrm{GHz}$ & $9.4 \times 10^{18} \sim 2.1 \times 10^{19}$ & Radar and experimental communication \\
\hline
\end{tabular}

Table 3 Inflow condition of the OREX at $59.4 \mathrm{~km}^{18}$

\begin{tabular}{cccc}
\hline Velocity, $\mathrm{U}_{0}[\mathrm{~m} / \mathrm{s}]$ & Temperature, $\mathrm{T}_{0}[\mathrm{~K}]$ & Wall Temperature, $\mathrm{T}_{w}[\mathrm{~K}]$ & Air density, $\mathrm{P}_{0}[\mathrm{~Pa}]$ \\
\hline 5561.6 & 248.12 & 1519 & 23.60 \\
\hline
\end{tabular}




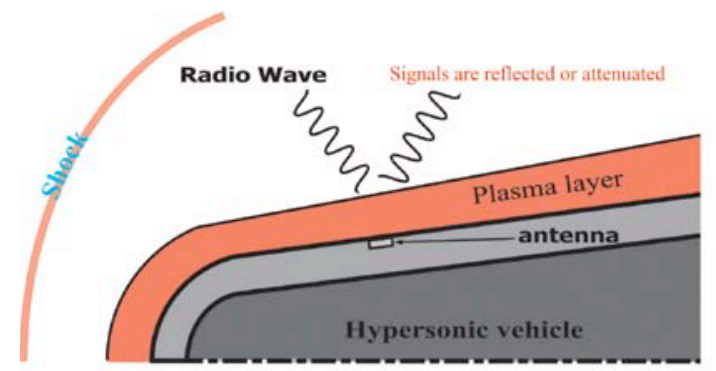

(a)

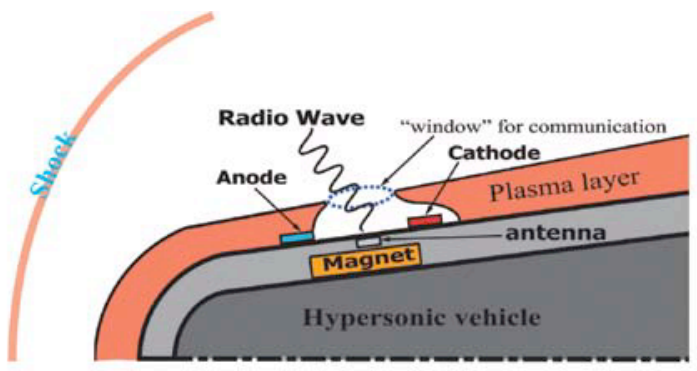

(b)

Figure 1. (a) A schematic of a hypersonic vehicle with approximate plasma layer around the vehicle. In this case, the radio waves are reflected or attenuated against the plasma layer. (b) A schematic of a hypersonic vehicle with approximate plasma layer around the vehicle with an ExB layer mitigation scheme. The applied ExB layer manipulates the plasma layer and creates a "window" for communication through the plasma layer.

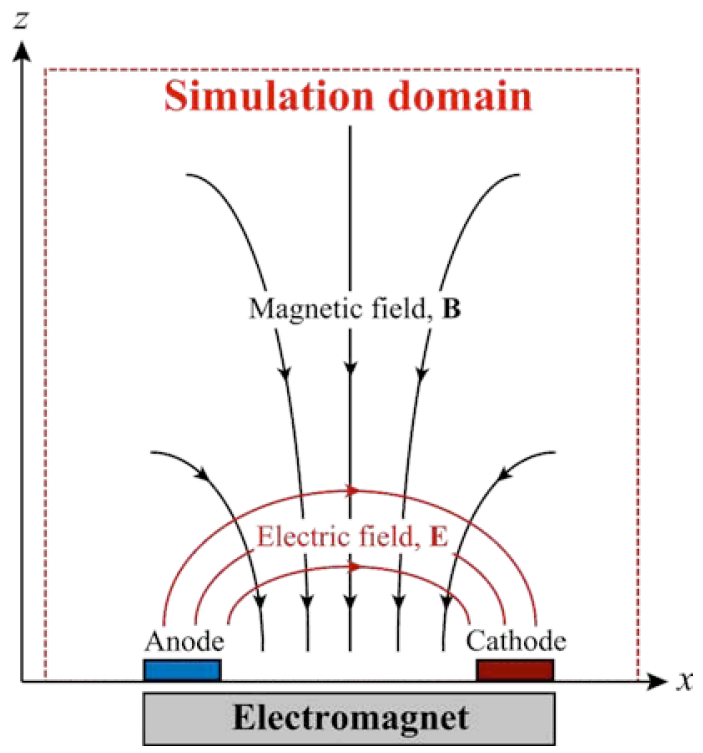

Figure 2. A schematic of the two-dimensional magnetic field model of the applied ExB layer. 


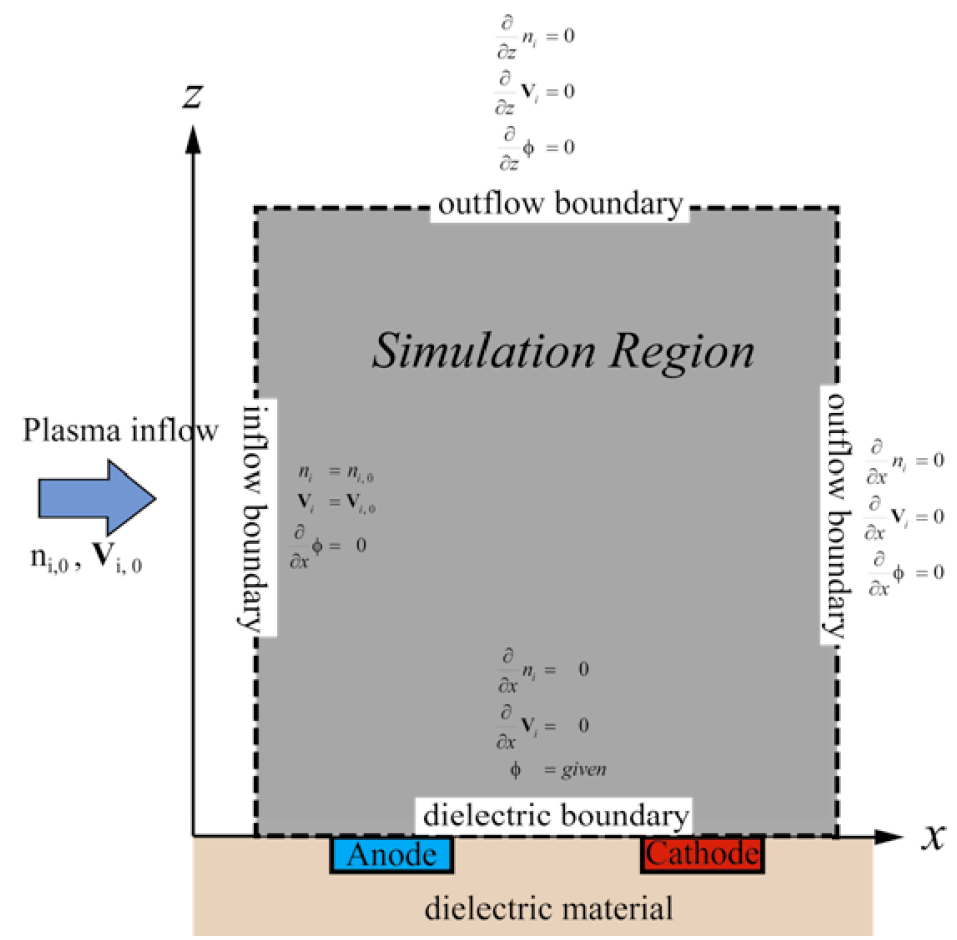

Figure 3. A schematic of the two-dimensional magnetic field model of the applied ExB layer.

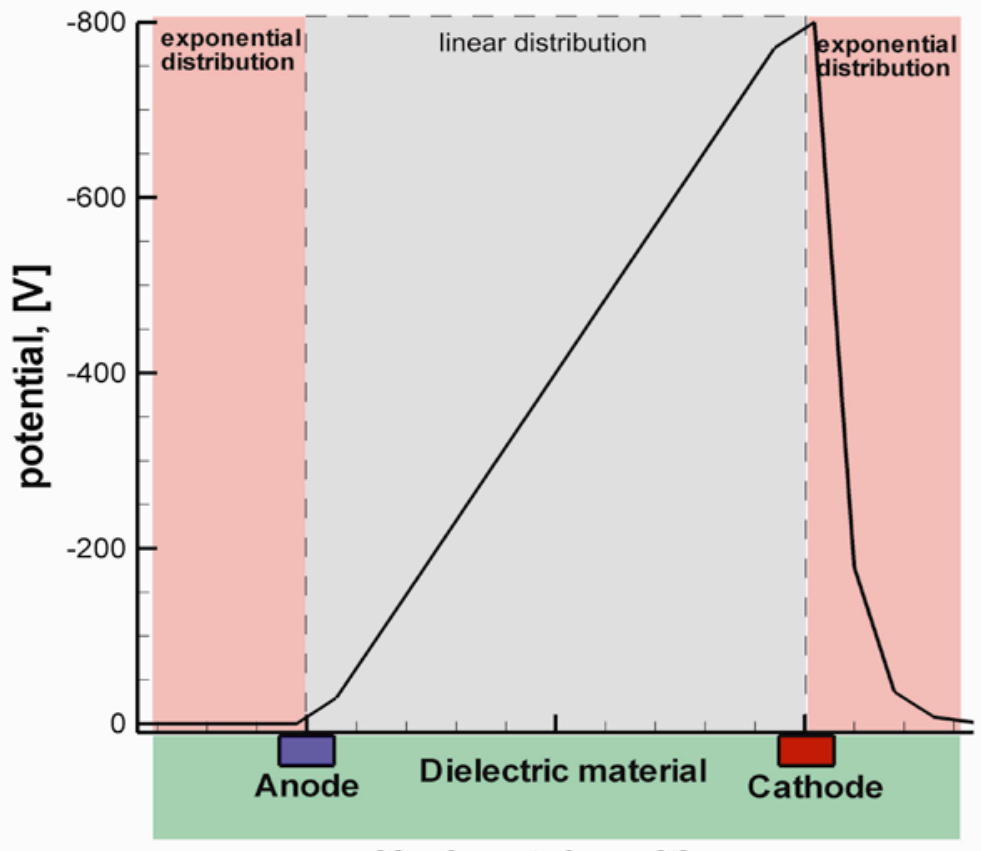

Horizontal position

Figure 4. A schematic of the two-dimensional magnetic field model of the applied ExB layer. 


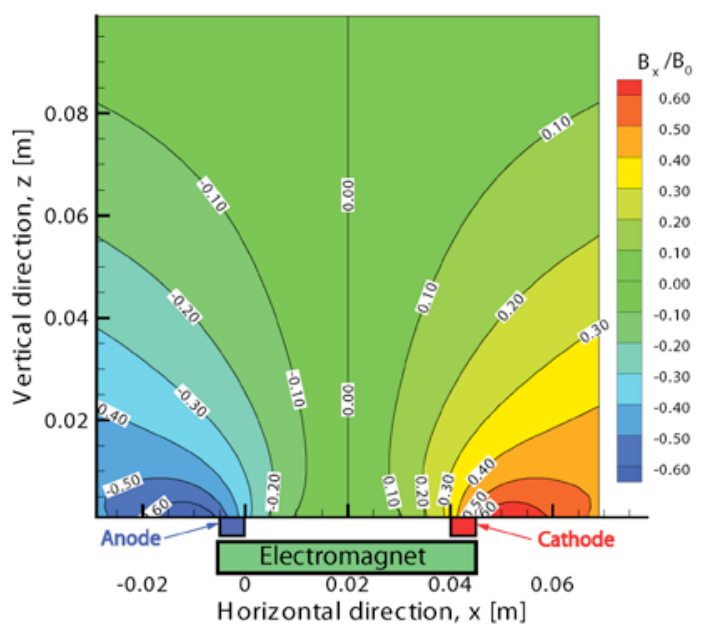

(a)

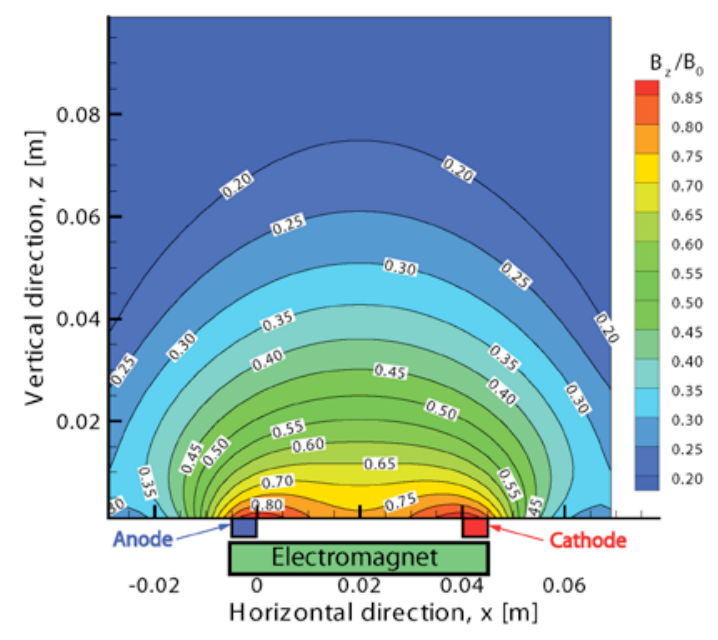

(b)

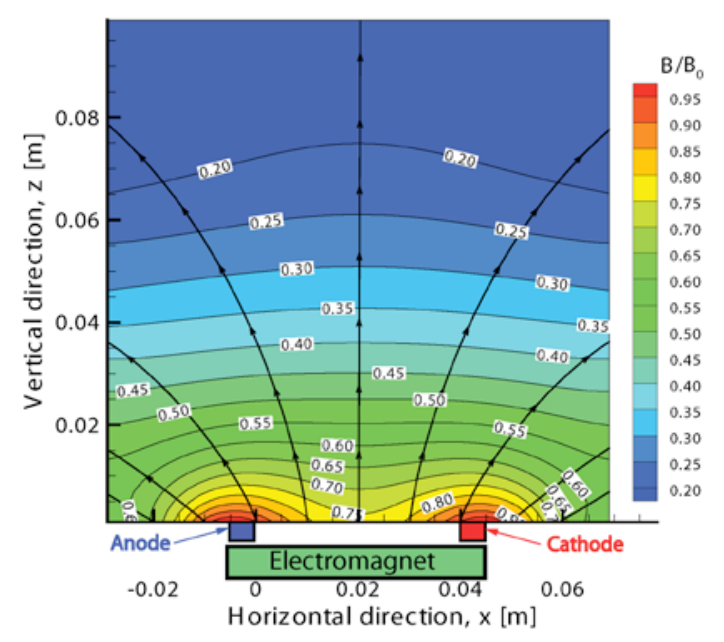

(c)

Figure 5. The applied magnetic field configuration which is normalized by the maximum magnetic field strength, $\mathbf{B}_{0}$.

(a) Horizontal direction magnetic field configuration, $\mathbf{B}_{\mathrm{x}} / \mathbf{B}_{0}$.

(b) Vertical direction magnetic field configuration, $B_{z} / B_{0}$.

(c) Normalized magnetic field strength configuration with the magnetic field lines. 


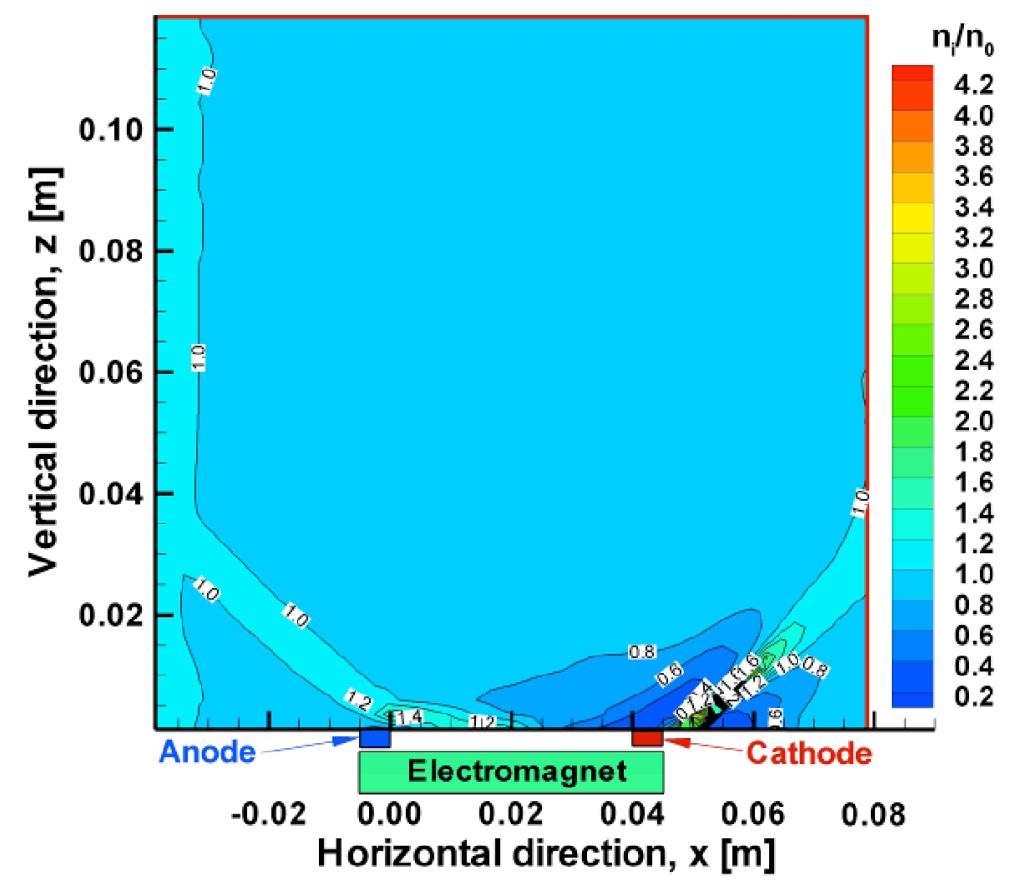

Figure 6. A distribution of plasma density reduction. The initial plasma density, $n_{0}$ is $4.2 \times 10^{16} \mathrm{~m}^{-3}$ and the initial neutral pressure is $1 \mathrm{mTorr}$. A $\mathbf{- 8 0 0} \mathrm{V}$ potential is applied with $0.07 \mathrm{~T}$ magnetic-field strength.

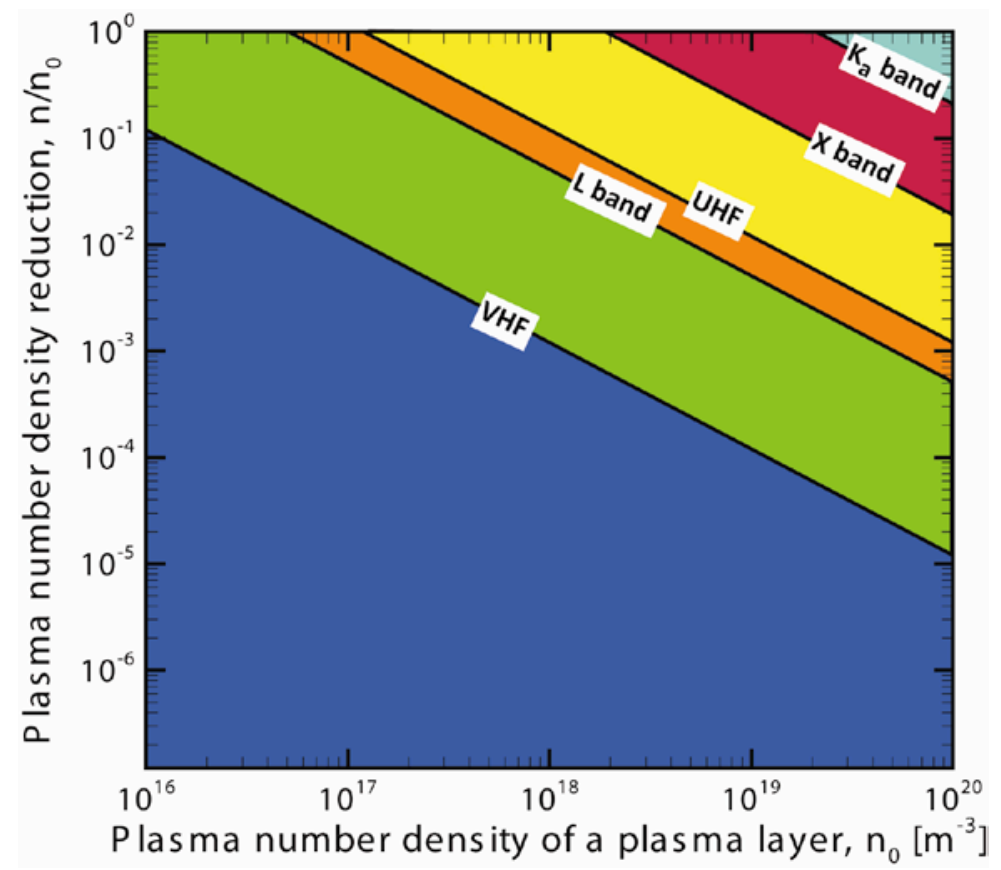

Figure 7. Required plasma-density reduction for several radio-wave frequencies. 


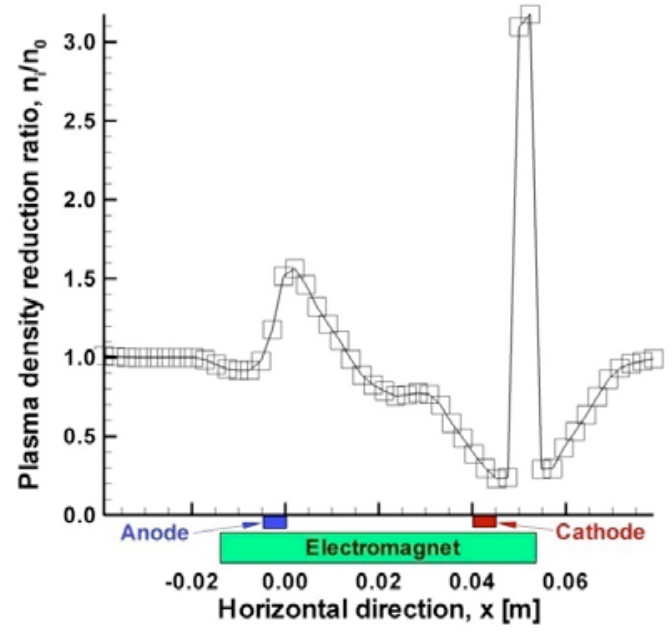

(a) $\mathrm{z}=0.0 \mathrm{~cm}$

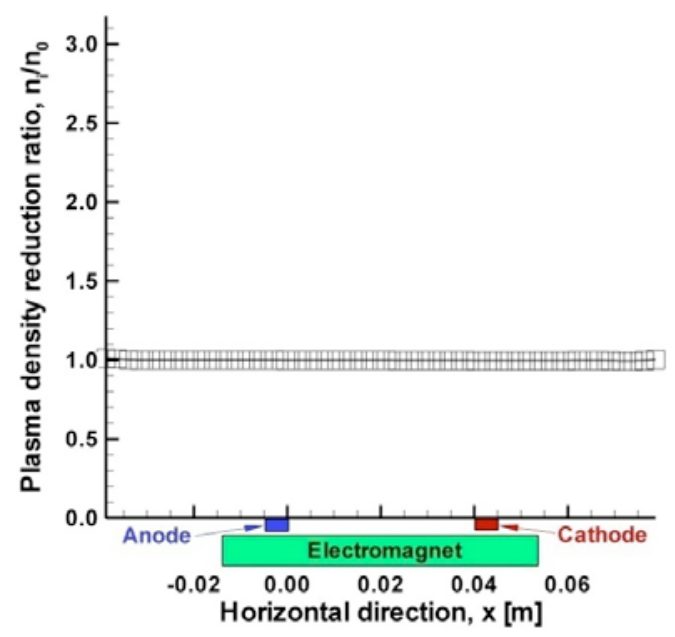

(c) $\mathrm{z}=4.0 \mathrm{~cm}$

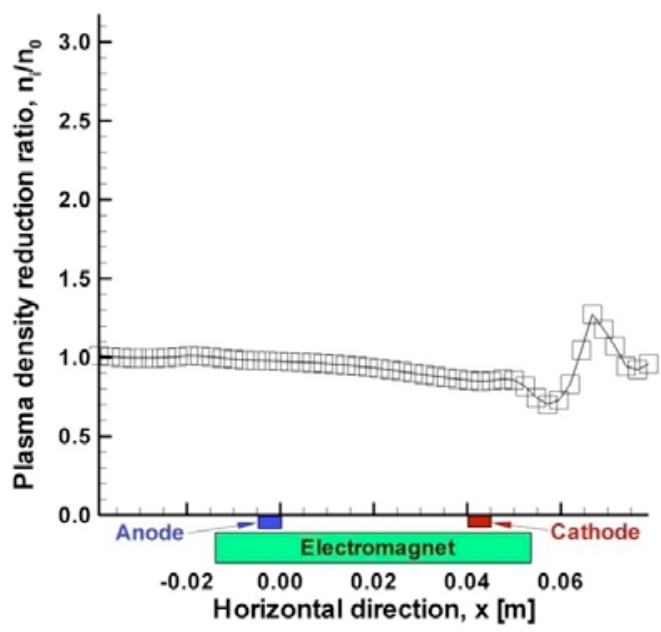

(b) $\mathrm{z}=2.0 \mathrm{~cm}$

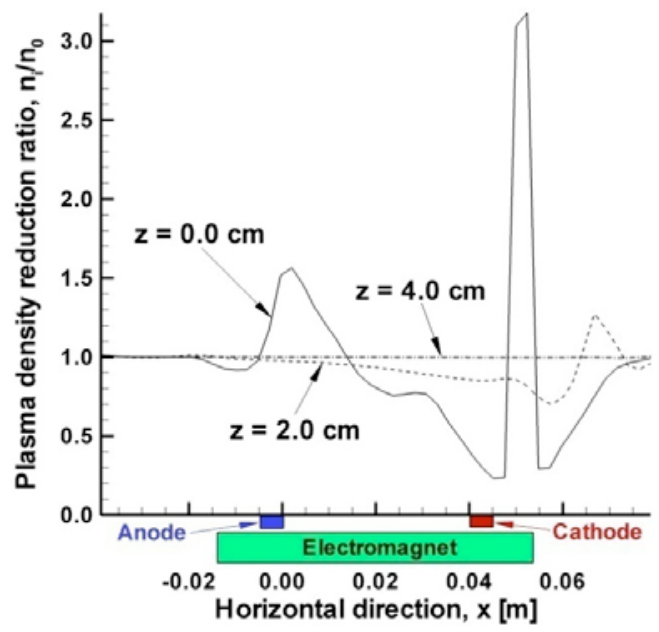

(d) $\mathrm{z}=0.0,2.0$ and $4.0 \mathrm{~cm}$

Figure 8. The distributions of the plasma density reduction at several vertical positions: $\mathrm{z}=0.0 \mathrm{~cm}, 2.0 \mathrm{~cm}$, and $4.0 \mathrm{~cm}$. The initial plasma density, $\mathrm{n}_{0}$ is $4.2 \times 10^{16} \mathrm{~m}^{-3}$ and the initial neutral pressure is $1 \mathrm{mTorr}$. A -800 V potential drop is applied with $0.07 \mathrm{~T}$ magnetic-field strength. 


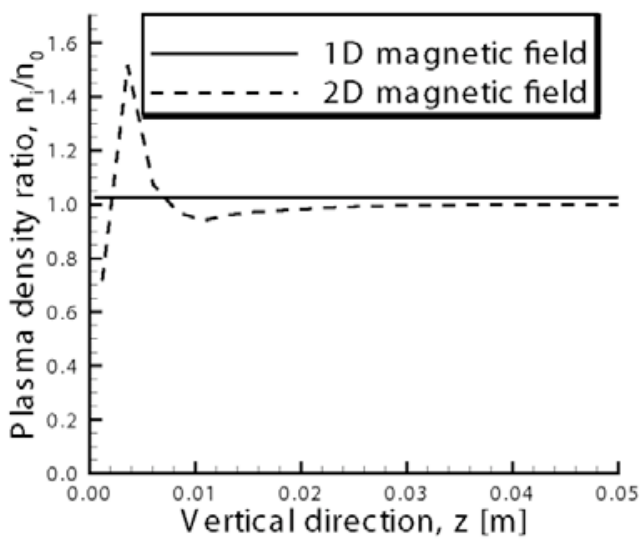

(a) $x=0.1 \mathrm{~cm}$

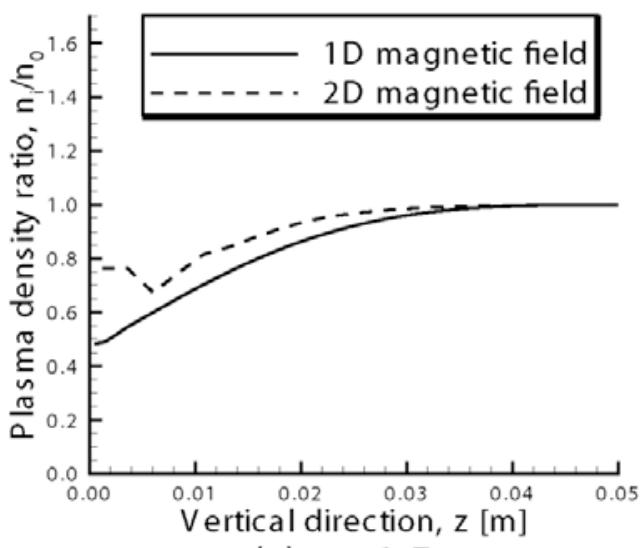

(c) $x=2.7 \mathrm{~cm}$

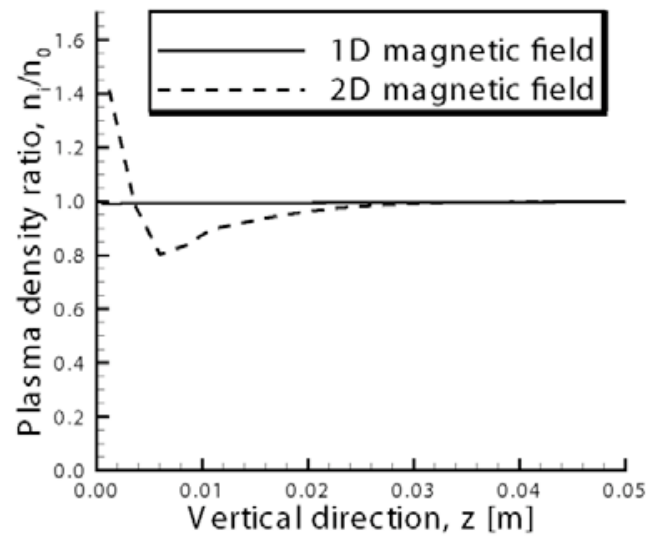

(b) $x=1.4 \mathrm{~cm}$

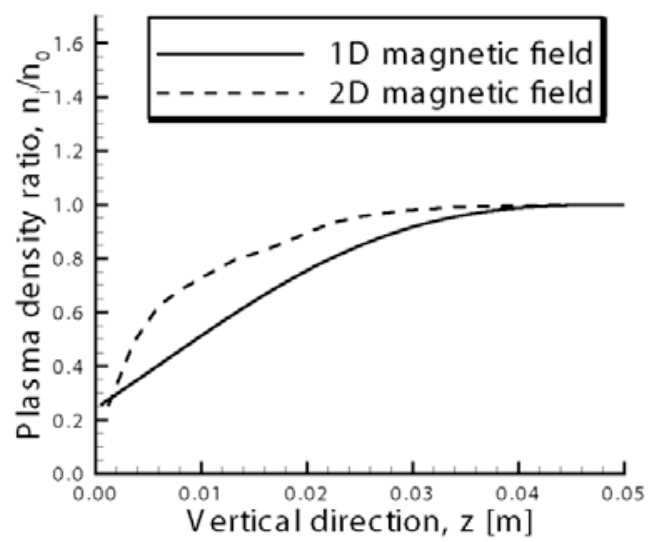

(d) $x=4.0 \mathrm{~cm}$

Figure 9. The distributions of the plasma density reduction with the one-dimensional magnetic field and two-dimensional magnetic field at several horizontal positions. The anode and cathode are located at $x=0.0$ $\mathrm{cm}$ and $4.0 \mathrm{~cm}$, respectively. The initial plasma density, $n_{0}$ is $4.2 \times 10^{16} \mathrm{~m}^{-3}$ and the initial neutral pressure is $1 \mathrm{mTorr}$. A -800 V potential drop is applied with $0.07 \mathrm{~T}$ magnetic-field strength. 


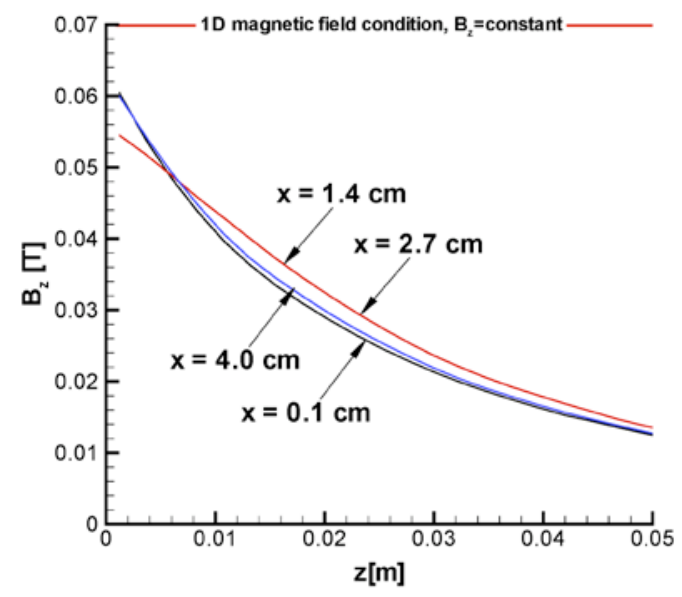

(a)

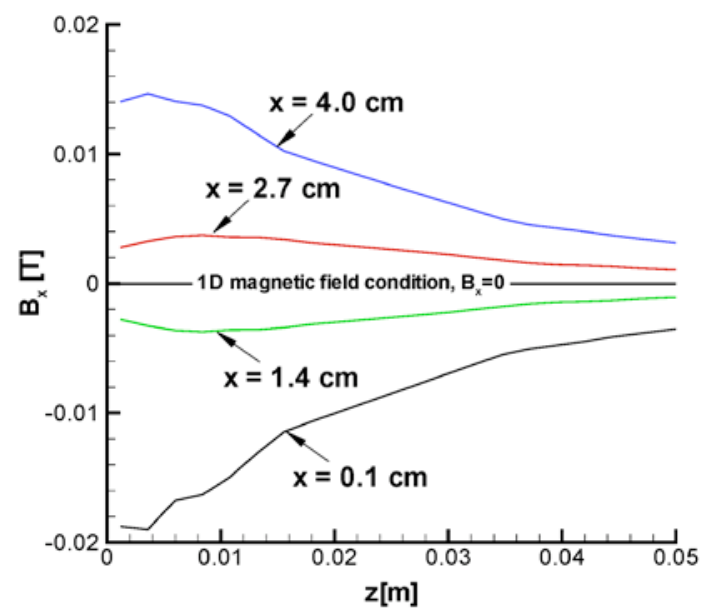

(b)

Figure 10. Magnetic field distributions for the 1D magnetic field model and measured data for the $B=0.07$ T case: (a) z-direction magnetic field strength, $B z$ (b)x-direction magnetic field strength, $B x$.

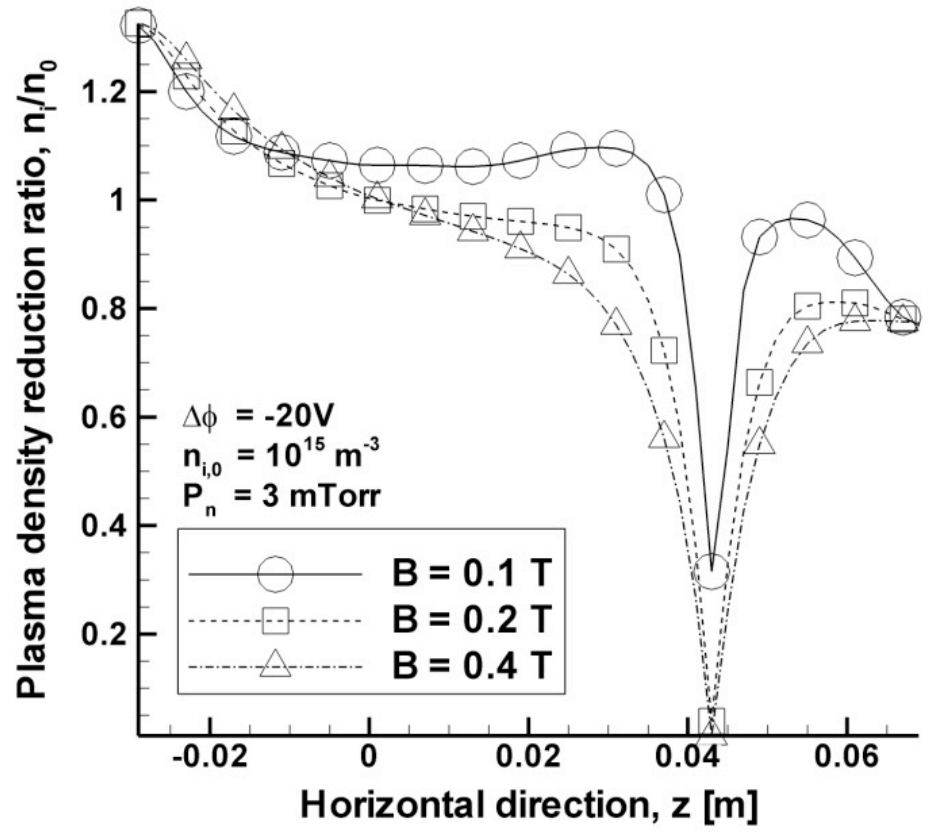

Figure 11. The plasma density reduction along the x-direction for several magnetic field strengths. 


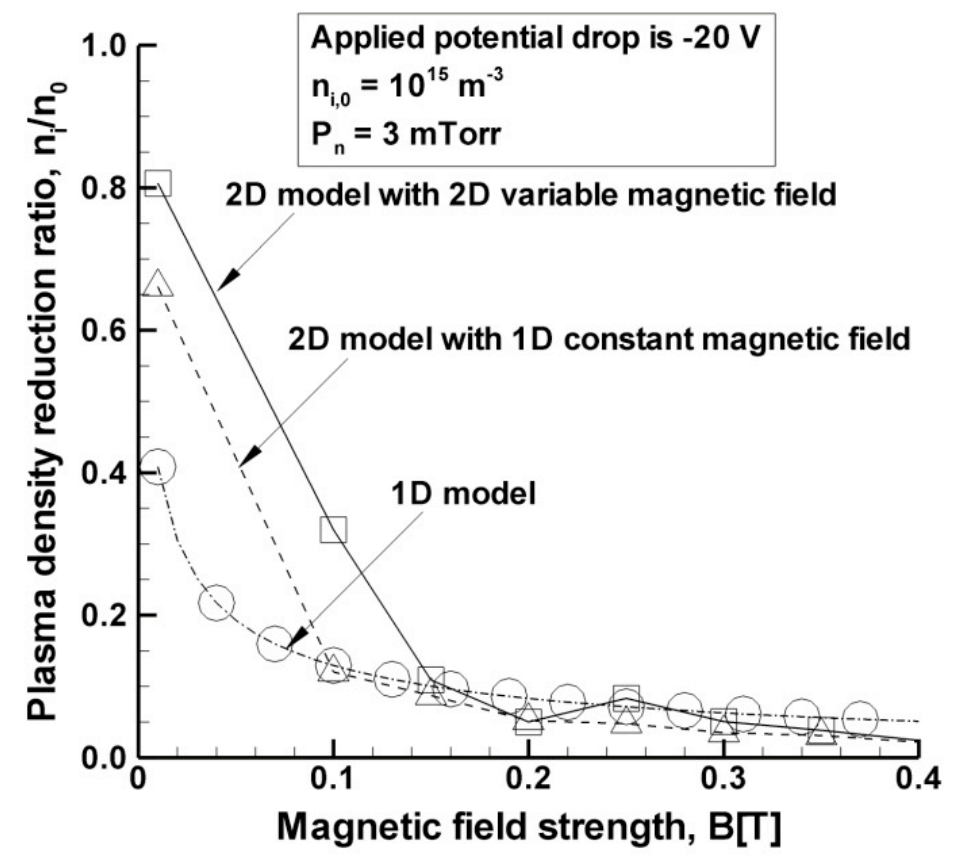

Figure 12. The plasma density reduction at the $z=4.0 \mathrm{~cm}$ position as a function of magnetic field strength.

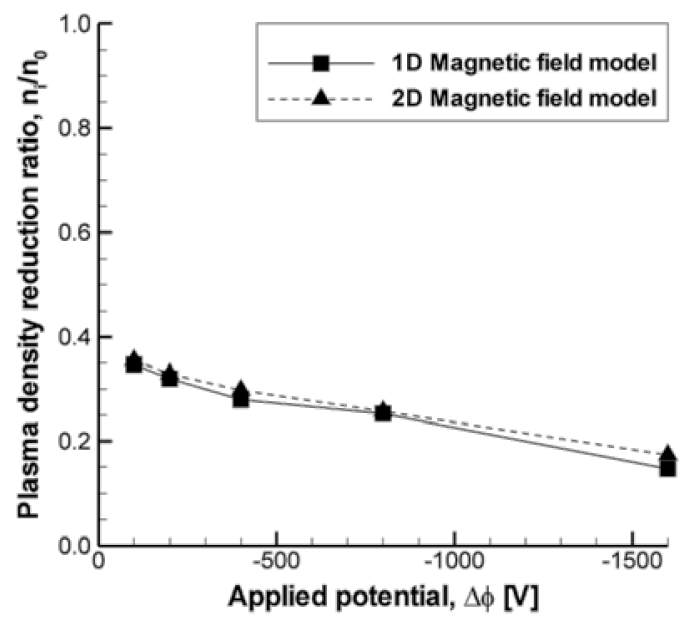

(a)

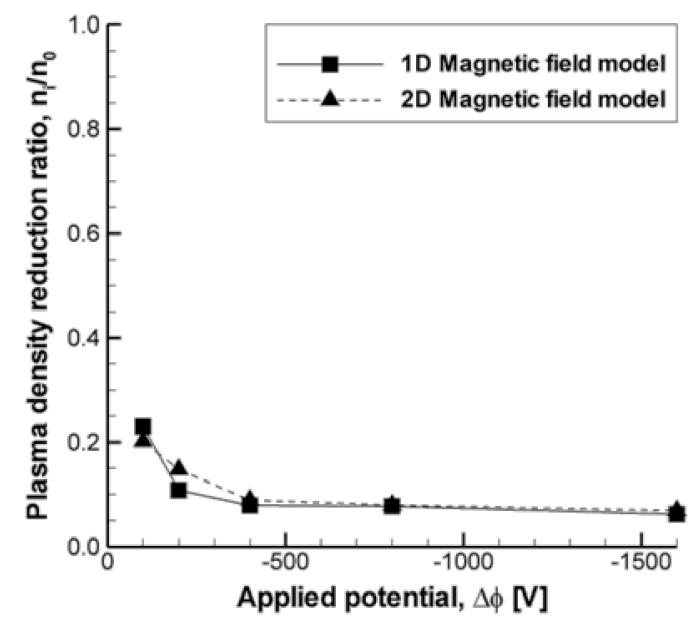

(b)

Figure 13. The plasma density reduction variation with applied potential drop

(a) The initial plasma density, $n_{0}$ is $4.2 \times 10^{16} \mathrm{~m}^{-3}$ and the initial neutral pressure is $1 \mathrm{mTorr}$. A -800 V potential drop is applied with $0.07 \mathrm{~T}$ magnetic field strength.

(b) The initial plasma density, $n_{0}$ is $2.4 \times 10^{16} \mathrm{~m}^{-3}$ and the initial neutral pressure is $150 \mathrm{mTorr}$. A -100 V potential drop is applied with $0.035 \mathrm{~T}$ magnetic field strength. 


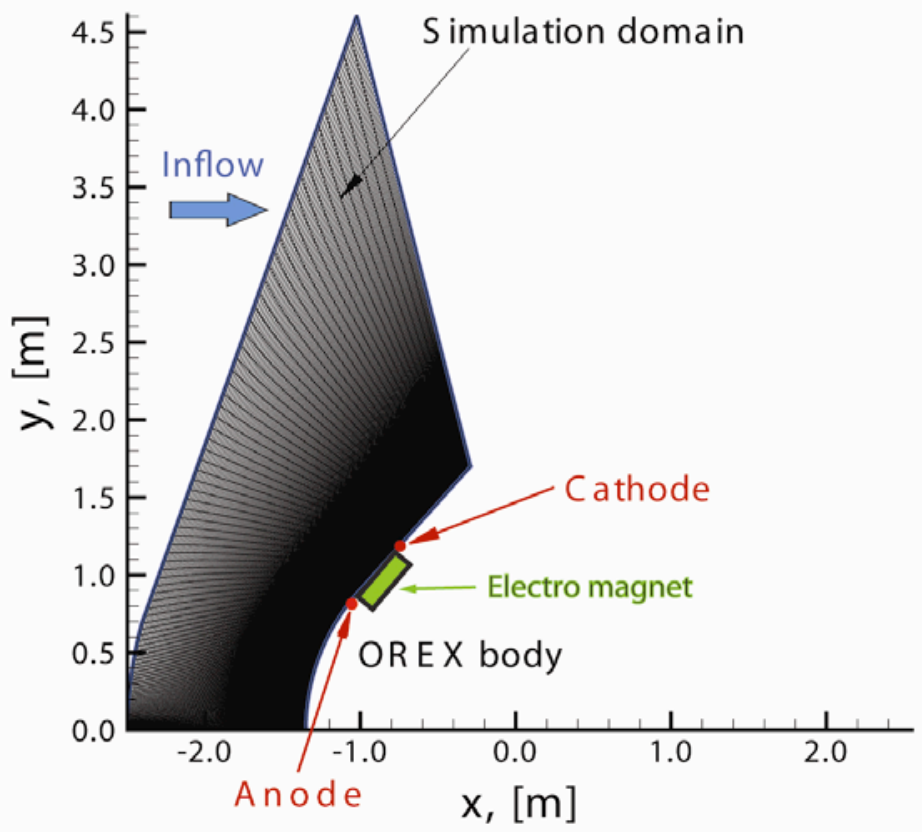

Figure 14. The simulation geometry and mesh for the OREX re-entry vehicle.
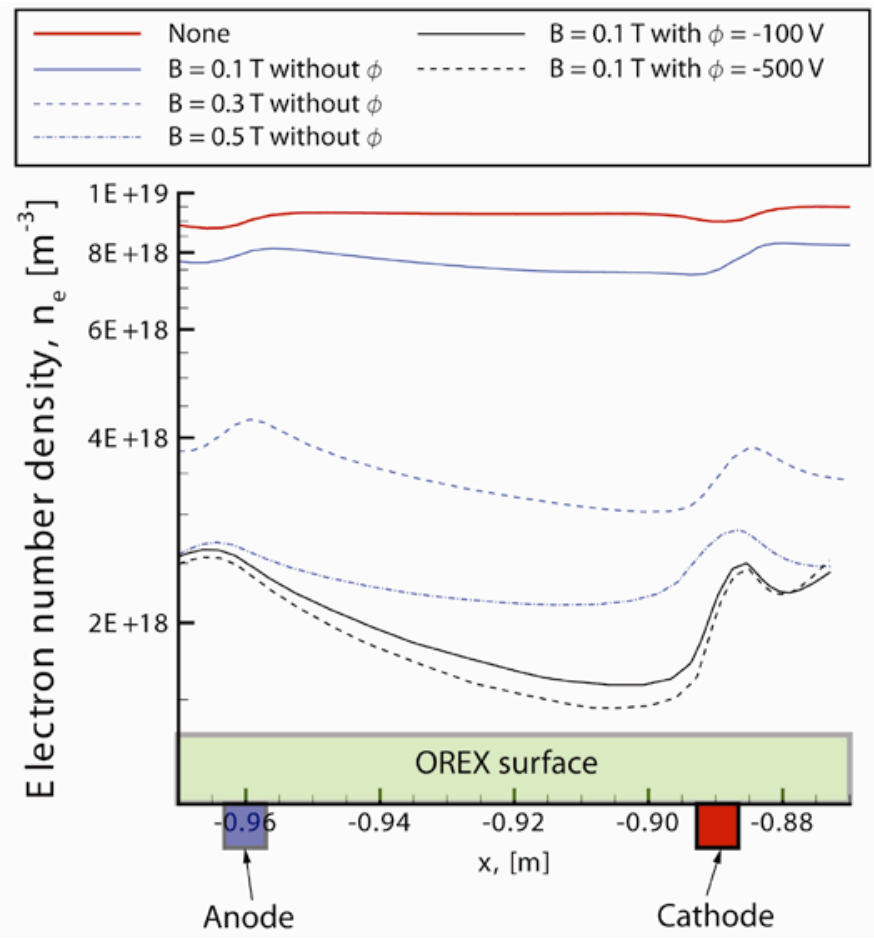

Figure 15. The electron number density distribution near the surface of OREX for several operational conditions of an ExB mitigation scheme.

American Institute of Aeronautics and Astronautics 092407 


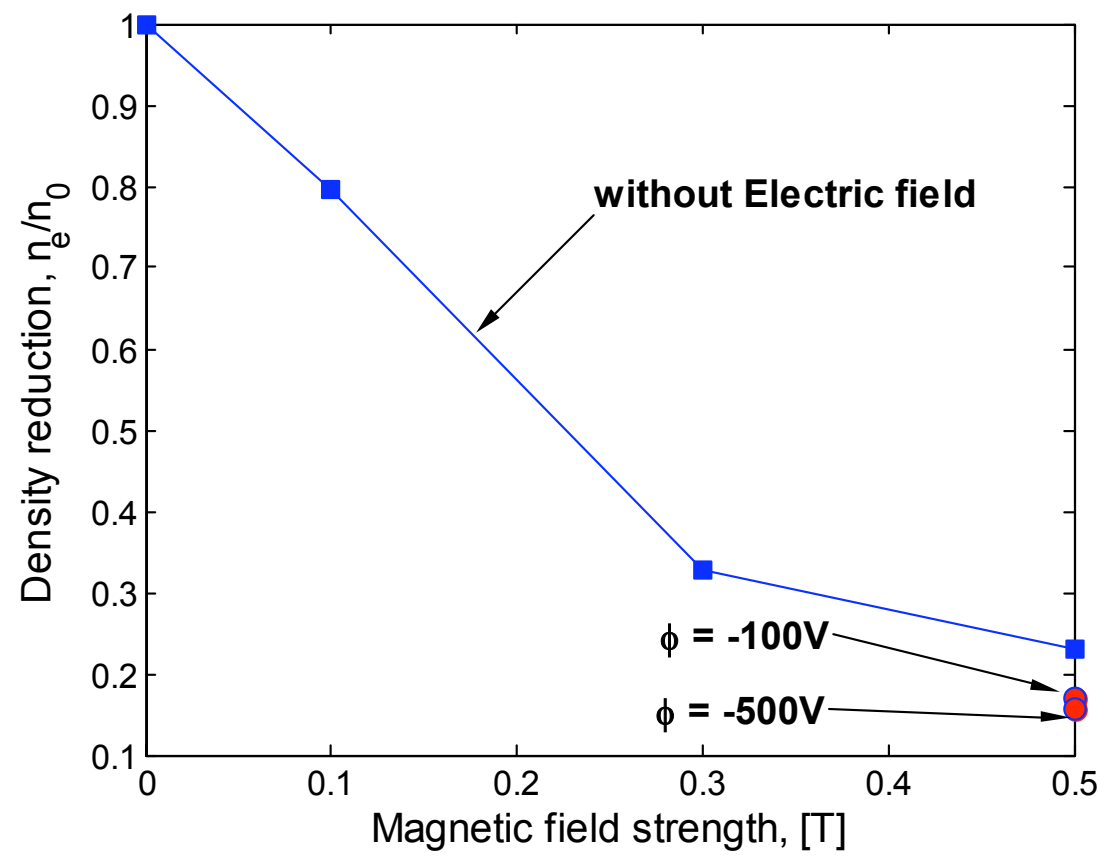

Figure 16. The maximum plasma density reduction by an ExB layer in a hypersonic flight condition. 